\title{
Psychodramatische-Selbsterfahrung im Gruppen- und Einzelsetting
}

\author{
Eine Reise zu sich selbst
}

\author{
Sabine Kern
}

Angenommen: 20. Januar 2022 / Online publiziert: 16. Februar 2022

(C) Der/die Autor(en) 2022

Zusammenfassung Dieser Artikel der Zeitschrift Psychodrama und Soziometrie beschäftigt sich mit psychodramatischen Arrangements, die im Einzel- und Gruppensetting für das Vorankommen im Selbsterfahrungsprozess hilfreich sein können. $\mathrm{Zu}$ Beginn wird auf Unterschiede zwischen Selbsterfahrung und Psychotherapie eingegangen. Die Voraussetzungen für eine gewinnbringende und sichere Reise zum Selbst, wie etwa eine gute therapeutische Beziehung, Gruppenkohäsion und klare Gruppenregeln, werden angeführt. Danach werden Arrangements vorgestellt, die zum besseren Verständnis der Beziehung zum Selbst beitragen sollen und psychodramatische Aufgabenstellungen präsentiert, die unterstützen, mögliche belastende Erfahrungen aus der Kindheit oder Jugend aufzuspüren. Ebenso wird auf psychodramatische Übungen eingegangen, die rigide Beziehungsmuster unter die Lupe nehmen. Zuletzt wird erörtert, welche wichtige Funktion Rollenspiele in der Selbsterfahrung einnehmen. Ein Resümee schließt den Artikel ab.

Schlüsselwörter Psychodrama - Selbsterfahrung · Arrangements · Einzel- und Gruppensetting

\footnotetext{
Sabine Kern $(\bowtie)$

Dürergasse 14-16, 1060 Wien, Österreich

E-Mail: sabine.kern@psychodramazentrum.at

Danube University Krems University for Continuing Education, Dr.-Karl-Dorrek-Straße 30, 3500 Krems an der Donau, Österreich
} 


\title{
Psychodramatic self-awareness in group and individual settings
}

A journey to yourself.

\begin{abstract}
This article in the Zeitschrift für Psychodrama and Soziometrie deals with psychodramatic arrangements that can be helpful in individual and group settings for progressing in the self-awareness process. At the beginning, the differences between self-awareness and psychotherapy are discussed. The prerequisites for a profitable and safe journey to the self, such as a good therapeutic relationship, group coherence and clear group rules, are listed. Afterwards, arrangements are presented that should contribute to a better understanding of the relationship to the self and psychodramatic tasks are presented, which support to track down possible stressful experiences from childhood or adolescence. Psychodramatic exercises are also discussed, which take a close look at rigid relationship patterns. Finally, it is discussed what important function role plays play in self-experience. A summary concludes the article.
\end{abstract}

Keywords Psychodrama $\cdot$ Self-awareness $\cdot$ Arrangements $\cdot$ Individual and group setting

Seit vielen Jahren biete ich psychodramatische Selbsterfahrung sowohl im Einzelals auch im Gruppensetting an. Die Personen, die sich diesem Prozess unterziehen sind einerseits Studierende, die eine Ausbildung zum*r Psychodramatherapeut*in absolvieren, andererseits Menschen, die sich im Rahmen einer Selbsterfahrungsgruppe mit sich selbst und ihren Beziehungsmustern auseinandersetzen wollen. Sie begeben sich dabei auf eine Reise zu den Höhen und Tiefen ihrer Seelenlandschaft. Als Reiseleiterin unterstütze ich sie, zu erfassen, welche Rollen sie innehaben, welche Eigenschaften sie mit Stolz erfüllen und welche lieber geheim gehalten werden. Es wird erforscht, welche Werte, Wünsche und Bedürfnisse das eigene Handeln leiten und wie man mehr Regie über das eigene Leben erlangen kann.

In diesem Artikel werden einige Arrangements vorgestellt, die Selbsterfahrungsprozesse anstoßen können. Manchmal können sie Teil einer protagonist*innenzentrierten Arbeit sein, manchmal auch als Erwärmung für ein anschließendes Protagonist*innenspiel eines*r Teilnehmer*in dienen. Die meisten Aufgabenstellungen sind sowohl im Einzel- als auch im Gruppensetting einsatzbar. Bei der Vorstellung der Arrangements wird beispielhaft immer nur eine Form beschrieben.

\section{Unterscheidung von Selbsterfahrung und Psychotherapie}

Eine besondere Fähigkeit von Menschen ist, sich selbst und das eigene Handeln zu reflektieren. Dieser Blick auf das eigene Selbst steht im Fokus von Selbsterfahrung, die der bewussten und gesteuerten Selbstentwicklung dient. Ziel ist es, die Kenntnis über die eigene Persönlichkeitsstruktur zu erhöhen und dysfunktionale Rollenkonfigurationen zu entlarven und zu verändern (Mösler und Poppek 2016, S. 19). Dazu gehört, sich der eigenen Stärken und Schwächen bewusst zu werden, die eigenen Leitmotive, Werte und verinnerlichten Normen kennen zu lernen und sich mit prä- 
genden Lebensereignissen auseinanderzusetzen. Das Bewusstwerden der eigenen Persönlichkeitsfacetten und Handlungsmuster soll bewirken, dass man nicht ständig in die gleichen Fallen tappt, nicht durch unerreichbare Ziele geleitet wird, weniger verletzbar ist und dadurch ein selbstbestimmtes Leben führen kann.

Im Gegensatz dazu stellt Psychotherapie eine Heilbehandlung dar, bei der die Verringerung von leidvollen Zuständen und störungsspezifischen Symptomen im Vordergrund steht. Während bei der Psychotherapie unter anderem eine Stabilisierung angestrebt wird und Sicherheit durch klare Strukturierung geboten wird, wird in der Selbsterfahrung Altbewährtes in Frage und Gewohntes teilweise auf den Kopf gestellt. Die Komfortzone emotionaler Stabilität (Brüderl et al. 2021, S. 30) muss mitunter verlassen, Verunsicherungen ertragen und den eigenen Ängsten ins Auge geblickt werden.

\section{Reisevorbereitungen}

Wie bereits erwähnt, kann es im Laufe der Selbsterfahrung aufgrund des Verlassens des vertrauten Terrains zu einer Destabilisierung kommen. Deshalb müssen Expeditionsteilnehmer*innen sich bewusst sein, dass die Reise auch Unsicherheiten und Beschwerlichkeiten mit sich bringen kann. Eine gehörige Portion an Motivation und Mut darf deshalb im Reisegepäck nicht fehlen. Es ist nicht ohne, die eigenen dunklen Seiten anderen und dem*der Gruppenleiter*in zu offenbaren oder sich dysfunktionalen Rollenkonfiguration zu stellen.

Damit man sich diesen Herausforderungen mit Engagement und Freude stellen kann, bedarf es einer guten Beziehung zum*zur Selbsterfahrungsleiter*in. Im Gruppensetting ist eine starke Gruppenkohäsion (Stadler und Kern 2010, S. 86) unerlässlich. Diese soll Halt und Vertrauen bieten, damit die teilnehmenden Personen sicher sein können, dass Berichte von schambesetzten Ereignissen wohlwollend aufgenommen und nicht später gegen sie verwendet werden.

\subsection{Regeln für ein gutes Gelingen der Reise}

Das Installieren und Einhalten von Gruppenregeln stellt für das Entwickeln von Vertrauen und das Erleben von Sicherheit in der Gruppe eine nicht zu missachtende Ausgangsbasis dar. Verschwiegenheit, einander ausreden lassen und ein wertschätzender Umgang miteinander bilden das Fundament einer gelingenden, gemeinsamen Reise. Ebenso muss vereinbart werden, wie anderen Gruppenteilnehmer*innen Rückmeldung gegeben werden soll, etwa in Ich-Botschaften, ohne zu analysieren und Ratschläge zu geben. Es soll gesichert sein, dass auch Wertschätzung entgegengebracht wird, wenn Schwächen gezeigt und negativ besetzte Anteile zu Tage treten.

\subsection{Aufbau einer tragfähigen Reisegruppe}

Wie oben beschrieben, ist für jede Gruppenreise in die Tiefen und Untiefen des Selbst der Aufbau einer tragfähigen Gruppenkohäsion unerlässlich. Dazu gibt es 
zahlreiche Arrangements, wie etwa aktionssoziometrische Übungen zum Kennenlernen oder Vorstellungsvarianten, wie zum Beispiel ,das Sich-Präsentieren über den Schlüsselbund“, die zum Ziel haben, etwas über die Mitreisenden zu erfahren. Der Gruppenzusammenhalt wird vor allem durch das Finden von Gemeinsamkeiten, das gegenseitige Zuhören, das Interesse aneinander und das gemeinsame Tun aufgebaut.

\subsubsection{Collage zur Vorstellung der Reisegesellschaft}

Zum besseren Kennenlernen der Teilnehmer*innen können persönliche Collagen erstellt werden, durch die mehr Einblick in die Lebensweisen, Wesenszüge und Vorlieben der Mitreisenden erlangt werden kann.

Den Teilnehmenden werden Papier, Illustrierte, Freecards, Stifte, Scheren und Klebstoff zur Verfügung gestellt. Die Aufgabenstellung ist, mit den vorhandenen Materialen ein Bild zu gestalten, das Facetten ihrer Persönlichkeit und Lebenssituation zeigt und vor allem widerspiegelt, was sie sich von dieser Selbsterfahrungsreise erwarten und welche Gebiete sie näher explorieren wollen.

Nach der Fertigstellung der Collagen, werden diese von den Teilnehmer*innen präsentiert, die Gruppenmitglieder werden aufgefordert, Fragen zu stellen. Zum Schluss werden bei einem gemeinsamen Blick auf alle Bilder, Gemeinsamkeiten und Unterschiede herausgearbeitet und die Stärken und Besonderheiten dieser Reisegesellschaft diskutiert.

Das Fotografieren oder Archivieren der Collagen ermöglicht, diese am Ende des Selbsterfahrungsprozesses noch einmal heranzuziehen und $\mathrm{zu}$ evaluieren, ob die Erwartungen erfüllt wurden.

\subsubsection{Eintrag in ein Reisekumpan*innenbuch}

Manchmal kommt auch ein adaptierter Fragebogen zum Einsatz, der in seinem Design und Aufbau einem Blatt eines Freundschaftsbuches ähnelt. Dieser beinhaltet Fragen, wie „Mein Lebensmotto lautet ...“, „Wenn ich ein Tier wäre, wäre ich ...“, „Wenn ich eine Zeitreise buchen könnte, würde ich in das Zeitalter ... reisen“. Die Teilnehmer*innen füllen den Fragebogen einzeln aus. In Kleingruppen oder im Plenum werden die Eintragungen vorgestellt. Auch hier soll das Finden von Gemeinsamkeiten nicht zu kurz kommen.

\section{Reise zum Selbst}

Im Psychodrama wird im Sinne Morenos davon ausgegangen, dass Rollen nicht aus dem Selbst entstehen, sondern dass sich das Selbst aus Rollen entwickelt (Petzold und Mathias 1982, S. 119). Das bedeutet, dass sich das Selbst einer Person nur aus Interaktionen mit anderen ausbilden kann, deren innerer Niederschlag sich zu einer Einheit verfestigt.

In den Worten von Schnabel, von Ameln und Stadler kann das Selbst in einem ,interaktionistischen Sinne als Summe der inneren und äußeren Reaktionsmuster auf jeweils situationsspezifische Anforderungen“ (Schnabel et al. 2019, S. 18) auf- 
gefasst werden. Schacht (2003, S. 35) unterscheidet das „Selbst als Objekt“, das im Sinne William James dem „Me“ entspricht und als Gesamtheit der inneren Repräsentationen der eigenen Person gesehen werden kann und dem „Selbst als Subjekt“, das „I“, das als Zusammenschluss ,,aller verfügbarer Kompetenzen“ (Schacht 2003, S. 36) der Handlungs- und Selbstregulation dient.

\subsection{Das Erforschen des Selbst anhand der Arbeit mit inneren Anteilen}

Das Psychodrama geht wie oben angeführt von einem pluralistischen Selbstkonzept aus (von Ameln et al. 2005, S. 228). Die Vorstellung, dass das Selbst aus vielen vernetzten Anteilen besteht, haben viele Vertreter*innen psychotherapeutischer Schulen aufgegriffen. Wie etwa Jochen Peichl (2011, S. $27 \mathrm{f}$.), der sich auf Watkins Ego-States beruft und mit seiner Ego-State-Therapie für die Behandlung von traumatisierten Personen wichtige Impulse gesetzt hat.

Nach Schnabel et al. (2019) werden innere Anteile als Rollen des kulturellen Atoms gesehen. „Sie sind Abbilder der eigenen Person und ihrer sozialen Bezogenheit, die in ihrer Gesamtheit die eigene Identität widerspiegeln, ausdrücken und gestalten. Innere Anteile sind daher sowohl Ergebnis als auch Ausgangspunkt der Persönlichkeitsentfaltung. Sie befinden sich untereinander, aber auch mit der äußeren sozialen Umwelt in andauernder wechselseitiger Beeinflussung" (Schnabel et al. 2019, S. 9).

Einer der bekanntesten Vertreter der Arbeit mit inneren Anteilen, die er als ,,innere Teamarbeit" publik gemacht hat, ist Schulz von Thun. Sein Modell ist für die psychodramatische Arbeit mit unterschiedlichen Rollenkonfigurationen sehr geeignet.

Schulz von Thun (1998 zitiert nach von Ameln et al. 2005, S. 228f.) skizziert das Selbst als ein Konglomerat von inneren Anteilen - ,eine Vorstellung, die er als innere Pluralität, innere Uneinigkeit, als inneren Dialog/Streit oder als innere Gruppendynamik bezeichnet" (von Ameln et al. 2005, S. 228). Durch die Personifizierung der inneren Anteile, wie etwa „der Kritiker“, „,der Angsthase“ oder „die Besserwisserin“, wird es leichter möglich mit diesen in Kommunikation zu treten und zu verhandeln sowie mit ihnen einen Rollenwechsel vorzunehmen. Auch innere Rollenkonflikte werden dadurch gut sichtbar.

\subsubsection{Mit Hilfe des ,inneren Teams“ neue Wege erproben}

Das Gefühl „Sich selbst im Wege zu stehen“ kann häufig mit einer besonders starken Ausprägung eines inneren Anteils in Zusammenhang stehen, wie etwa „dem inneren Kritiker", der zum Beispiel aufgrund seines perfektionistischen Anspruchs verhindert, dass eine schriftliche Abschlussarbeit fertig gestellt wird.

Eine Möglichkeit des Einsatzes des ,inneren Teams“ ist folgende: Ein Kreis wird im Sinne eines kulturellen Atoms auf ein Blatt Papier gezeichnet. Maud Bermann und Hermann Küster (2019, S. 105) schlagen vor, vorher aufzulisten, welche inneren Teammitglieder dem*der Klient*in überhaupt bekannt sind, wie ,der Antreiber“, „das Kind“ oder „,die Harmoniesuchende“. Danach soll eine für den Selbsterfahrungsprozess wichtige Frage formuliert werden, wie zum Beispiel „Welche Anteile 
haben bei inneren Teamsitzungen häufig das Sagen?" Nun soll der*die Klient*in jene inneren Teammitglieder aufzeichnen oder deren Bezeichnung aufschreiben, die sich melden, wenn Problemlagen anstehen. Je stärker diese ausgeprägt sind, umso größer soll deren Darstellung sein.

Im Anschluss soll der*die Klient*in, das Atom unter folgenden Prämissen betrachten: Wie zufrieden bin ich mit meinem ,,inneren Team“? Besteht ein Ungleichgewicht? Fehlen wichtige Mitglieder oder Antagonist*innen, die ein Gegenwicht zu besonders dominanten Teammitgliedern bilden könnten? Welche sind sichtbar, welche handeln im Verborgenen? Durch einen Rollenwechsel mit verschiedenen Teammitgliedern lässt sich in Erfahrung bringen, welchen besonderen Zweck die jeweiligen Anteile verfolgen, warum der eine so groß und der andere so klein ist. Ein weiterer Schritt wäre zu eruieren, was benötigt werden würde, damit ein besseres Gleichgewicht erzielt werden kann.

\subsubsection{Mit sich selbst in verschieden Lebensaltern in Kontakt treten}

Eine andere Form der inneren Team-Arbeit stellt das Einberufen von „Ichs“ in unterschiedlichen Lebensphasen zu einer Thematik dar. So kann zum Beispiel die Fragestellung „Wie kann ich mich motivieren, mehr Sport zu betreiben?“ im Austausch mit ein und derselben Person, die aber in verschiedenen Altersstufen vertreten ist, beantwortet werden.

\subsection{Das Erforschen des eigenen Selbstwert}

Das Selbstwertgefühl ist die Emotion, die entsteht, wenn eine Person über sich selbst, die eigenen Fähigkeiten und Handlungen nachdenkt und diese bewertet (Wikipedia 2021). Auch im Rahmen von Selbsterfahrung spielt der Selbstwert und wie dieser reguliert werden kann, eine große Rolle. Personen, die unter einem schwach ausgeprägten Selbstwert leiden, haben meist einen defizitorientieren Blick auf sich selbst, haben Angst, Fehler zu machen, überschätzen die Stärken ihres Gegenübers und sind oftmals leicht kränkbar (Brüderl et al. 2021, S. 125). Ein gutes Selbstwertgefühl hängt unter anderem von der Kenntnis der eigenen Stärken und einer liebevollen Selbstakzeptanz ab. Die nachfolgende Übung stellt unter Einbeziehung verschiedener Sinne eine Möglichkeit dar, das eigene Selbst mit Wohlwollen zu erforschen.

\subsubsection{Den Blick auf sich selbst richten}

Mit diesem Arrangement sollen auf achtsame und liebevolle Weise die eigenen Fähigkeiten, Eigenschaften, besondere Kompetenzen, aber auch Schwachstellen und Verletzlichkeiten dargestellt werden. Ziele dieser Übung sind, sich bewusst zu werden, wie groß die Bandbreite der eigenen Stärken ist und die eigene Selbstakzeptanz zu erhöhen.

Die Teilnehmer*innen gehen zu zweit zusammen. Eine*r legt sich auf ein dafür vorbereitetes Packpapier, die andere Person zeichnet ihre Silhouette nach. Die Position, ob etwa von der Seite oder frontal, kann selbst gewählt werden. Danach 
befasst sich jede mit ihrem eigenen Abbild. Nun gilt es, diese Körperdarstellung „mit Leben zu erfüllen“. Besondere Merkmale, wie etwa die großen blauen Augen oder ein offenes Herz für andere zu haben werden eingezeichnet, besondere Fertigkeiten, wie zum Beispiel gut und mit Leidenschaft zu kochen werden in den Körperumriss hineingeschrieben. Aber auch nicht so positiv besetzte Eigenschaften, wie etwa die Neigung zu Prokrastination sollen hier Platz finden. Wichtig ist, dies mit viel Wertschätzung sich selbst gegenüber auszuführen. Manchmal kann es bei der Ausführung der Aufgabe hilfreich sein, in die Rolle eines*einer liebevollen Freund*in zu schlüpfen.

\subsubsection{Besichtigung der „Fünf Säulen der Identität“ nach Hilarion Petzold}

Hilarion Petzold war Psychodramatiker, bevor er sich der Integrativen Therapie zuwandte. 1993 entwickelte er das „Fünf Säulen Modell der Identität“, wobei er Identität als die einzigartige Persönlichkeitsstruktur eines Menschen definiert.

In seinem Modell geht Petzold (1993, S. 276) davon aus, dass die Identität durch fünf Pfeiler getragen wird. Diese sind: Leiblichkeit, soziale Beziehungen, Arbeit und Leistung, materielle Sicherheit sowie Werte und Ideale. Identitätskrisen sind häufig mit einem Wegbrechen einer dieser Säulen verbunden, wie zum Beispiel ein Jobverlust. Sind die restlichen Säulen aber solide gebaut, kann das Wegfallen eines Pfeilers kompensiert werden und eine emotionale Destabilisierung eingeschränkt werden.

Auch wenn das Modell schon etwas antiquiert wirken mag, eignet es sich dennoch sehr gut, um zu erkennen, ob eine Person über ausreichende Ressourcen verfügt, um auch in bewegten Zeiten nicht aus dem Gleichgewicht zu geraten.

Ziel dieser Aufgabenstellung ist, zu überlegen, ob die einzelnen Säulen so tragfähig sind, dass auch Krisen überstanden werden können oder ob bei dem ein oder anderen Pfeiler dringender Renovierungsbedarf besteht.

Dazu werden dem*der Klient*in zwei Blätter vorgelegt. Auf dem ist das Modell von Petzold mit der Bedeutung der Säulen abgebildet. Das andere Blatt bietet die Möglichkeit, die eigenen Befindlichkeiten in die Säulen einzutragen.

1. Säule Leiblichkeit Wie wohl fühle ich mich in meinem Körper? Stehen Körper, Seele und Kognition im Gleichgewicht? Wie fit und körperlich stark erlebe ich mich?

2. Säule Soziale Beziehungen Wie zufrieden bin ich mit meinem sozialen Atom? Kann ich Kraft aus meinen sozialen Beziehungen, meiner Partnerschaft oder/und meiner Familie schöpfen?

3. Säule Arbeit und Leistung Wie zufrieden bin ich mit meiner Erwerbs- und meiner unbezahlten Arbeit? Wieviel Anerkennung kann ich daraus beziehen? Kann ich meine Fähigkeiten in meiner Arbeit einbringen? Ist meine Work-Life-Balance im Gleichgewicht?

4. Säule Materielle Sicherheit Verfüge ich über ausreichende finanzielle Ressourcen, um ein gutes und entbehrungsarmes Leben führen zu können? Wie zufrieden 
bin ich mit meiner Wohnsituation? Habe ich finanzielle Ressourcen, um eventuelle Krisensituationen gut überstehen zu können?

5. Säule Werte und Ideale Welche Werte und ethische Normen sind für mich von Bedeutung? Wie sieht mein axiometrische Atom (siehe weiter unten) aus? Bin ich damit zufrieden oder sind manche Werte überholt?

Der oder die Klient*in wird gebeten sich mit diesen Fragen auseinanderzusetzen. Danach wird aus der Spiegelposition ein Blick auf die Tragfähigkeit der einzelnen Säulen gelegt. Welche sind besonders massiv und stabil, welche sind ausbaubedürftig? Welche Maßnahmen könnten ergriffen werden, damit die Säulen so tragfähig sind, dass sie auch bei starker Belastung, nicht zum Einsturz gebracht werden können.

\subsection{Exkursion ins axiometrische Atom}

Ein Arrangement, das bei der fünften Säule der Identität erwähnt wurde, ist das axiometrische Atom (vgl. Döller 2016), das sich mit der eigenen Weltanschauung, dem Wertesystem und gesellschaftlichen Normen auseinandersetzt. Durch dieses kann eruiert werden, wie Einstellungen und Werthaltungen die Lebensgestaltung beeinflussen und durch wen oder welche Ereignisse diese geprägt wurden.

Hierfür wird zum Beispiel auf der Raumbühne ein Kreis mit einem Seil gebildet. Dieses stellt das axiometrische Atom dar. Dann werden Symbole für die Einstellungen, Werte oder auch Lebensmottos gewählt. „Für mein Streben, alles mit Gelassenheit zu nehmen, wähle ich diese Buddha-Statue und für meine christliche Werthaltung dieses Bild von einem Erzengel“. Je bedeutender diese Lebenseinstellung ist, umso mehr steht sie im Mittelpunkt. Symbole, die am Rand platziert sind, sind von minderer Wichtigkeit. Aus der Spiegelposition am Bühnenrand wird besprochen, ob dieser Wertekanon für die Person so passt oder ob es Bedürfnis nach Veränderung gibt. Gibt es Einstellungen, die man innehat, weil sie der Familientradition entsprechen, wie zum Beispiel eine politische Partei zu favorisieren? Fühlt man sich durch diese bezüglich eigener Werte noch vertreten? Durch den Einsatz psychodramatischer Handlungs- und Bühnentechniken können Zusammenhänge zwischen Erfahrungen und Werthaltungen erkannt, Normen hinterfragt und Veränderungen vorgenommen werden.

\section{Zeitreise in die Vergangenheit}

Kaum ein*e Seelenerforscher*in kommt umhin, ein Zeitreise in die Vergangenheit und insbesondere in die Kindheit zu unternehmen. Dabei soll in Erfahrung gebracht werden, welche lebensgeschichtlichen Ereignisse besonders prägend waren und welche Botschaften von Eltern und Angehörigen dem Kind mit auf dem Weg gegeben wurden. Einige von ihnen haben noch heute Einfluss auf das eigene Erleben und Handeln. Manche bieten Orientierungshilfe, Sicherheit, u. v. m. und stellen sich damit als hilfreich dar, andere wiederum können sich als wahre Stolpersteine entpuppen. Einer Auseinandersetzung mit den Beziehungserfahrungen, die mit der Mutter, dem 
Vater und den Geschwistern gemacht wurden, soll im Selbsterfahrungsprozess viel Zeit und Raum gegeben werden. Denn dadurch kann der eigene Werdegang besser verstanden werden.

Mit welchen psychodramatischen Arrangements diese Auseinandersetzung unterstützt werden kann, wird in den nächsten Absätzen dargestellt.

\subsection{Im Zeitraffer das eigene Leben betrachten}

Will man schnell und kompakt einen Überblick über Meilensteine des Lebens erhalten, bewährt sich das Erstellen einer Lebensline. Dazu kann sowohl die Blatt-, die Tisch- als auch die Raumbühne genutzt werden. Dafür werden zum Beispiel mittels eines Koordinatensystems wichtige Ereignisse im Leben einer Person auf der x-Achse aufgelistet, auf der y-Achse werden die dazugehörigen Gefühlslagen angeführt. Ebenso kann mittels eines Seils die Lebensline des*der Klient*in auf der Raumbühne dargestellt werden. Mit Intermediär-Objekten werden wichtige Stationen im Werdegang des*der Protagonist*in symbolisiert. Der*die Klientin erhält damit eine Übersicht über das bisherige Leben und die damit verbundenen Emotionen und Herausforderungen.

Dieses Arrangement kann einen Ausgangspunkt für weiterführende Exkursionen bieten: Zum Beispiel einen stärken Fokus auf einen Lebensabschnitt setzen, weil bestimmte Kränkungen noch nicht verarbeitet worden sind oder es wird ein Blick in die Zukunft geworfen, wie etwa die Lebenslinie optimaler Weise weiterverlaufen könnte.

\subsection{Dem Sozialen Atom der Kindheit einen Besuch abstatten}

Mit dieser Übung sollen Werte und Aufträge, die von Bezugspersonen an die Folgegeneration weitergegeben wurden, reflektiert werden.

Dazu werden die Menschen auf die Bühne eingeladen, die für den*die Protagonist*in in der Kindheit von besonderer Bedeutung waren. Für diese werden HilfsIche gewählt. Für sich selbst wählt die Person ein Double. Rund um das Double werden die Bezugspersonen (Hilfs-Iche) des*der Protagonistin positioniert. Der Abstand zum*r Protagonist*in soll die emotionale Nähe widerspiegeln, die derzeit zu dieser Bezugsperson erlebt wird, auch wenn diese unter Umständen schon verstorben ist. Im Anschluss werden die Hilfs-Iche eingerollt bzw. ein Rollenwechsel vollzogen.

Herausgefunden werden soll: Wie beschreiben die Bezugspersonen ihre Beziehung zum*r Protagonist*in? Welche Werte oder Lebensweisheiten haben die Personen dem/der Protagonist*in mitgegeben?

Danach wird geklärt, welche Botschaften und Regeln gerne angenommen werden. Wofür der*die Protagonist*in dankbar ist, für welche kein Bedarf mehr besteht oder welche als belastend erlebt werden. Hier kann sich ein Dialog zwischen dem*der Protagonist*in und den Bezugspersonen entwickeln. Werte und Normen, die nicht mehr gebraucht werden, können symbolisch zurückgegeben werden. 


\subsection{Den Familienstammbaum erkunden}

Einigen Aufschluss über die Einflüsse, die das heutige Leben prägen und auch das Verständnis für die Lebensbedingungen der vorangegangenen Generationen, kann das Aufstellen oder Aufzeichnen eines Familienstammbaums geben. Hierfür können zum Beispiel - wenn vorhanden - Fotos herangezogen werden, die in Form eines Stammbaums auf ein großes Blatt Papier geklebt werden. Die Lebensumstände, die Eigenschaften der einzelnen Vorfahren und die damaligen soziokulturellen und politischen Begebenheiten können neben der Abbildung geschrieben werden.

Beim Betrachten des Stammbaumes kann überlegt werden, welche Lebensverläufe, Schicksalsschläge, Wertesysteme noch Einfluss auf das heutige Leben des*der Klient*in haben. Was unterscheidet die eigene Lebensführung von der früherer Generationen?

\subsection{Post an die Herkunftsfamilie schicken oder einen Brief von ihr erhalten}

Um das eigene Sein so richtig erfassen zu können, ist eine vertiefte Beschäftigung mit den eigenen Eltern unumgänglich.

Ein Arrangement, das als Erwärmung zu einer vertieften Auseinandersetzung mit der Beziehung zu diesen engen Bezugspersonen dienen kann, ist einen Brief an sie zu verfassen. Die Gruppenteilnehmer*innen werden durch eine Imagination unterstützt, in sich zu gehen und zu überlegen, was sie ihren Eltern oder einem Elternteil sagen wollen. Im Anschluss werden sie aufgefordert, dies in Form eines Briefes an ihre Eltern niederzuschreiben mit dem Wissen, dass dieser nicht abgeschickt wird. In diesem können sie sich bedanken, Kränkungen ansprechen, Wünsche äußern, Anekdoten in Erinnerung rufen, Wertschätzung äußern, die Liebe und/oder auch die Wut zum Ausdruck bringen. Der Brief wird in ein Kuvert gesteckt und symbolisch abgeschickt.

Eine umgekehrte Variante dieser Übung ist, dass sich die Selbsterfahrungsteilnehmer*innen vorstellen, dass sie einen Brief von ihren Eltern oder einem Elternteil erhalten. Was wäre der Inhalt dieses Briefes. Würde in ihm Versöhnliches, Wertschätzendes, Liebevolles oder Bestärkendes zu lesen sein? Die Emotionen, die so ein Brief auslösen würde, kann im Zweier- oder Gruppengespräch ausgetauscht werden.

\section{In die Geheimnisse der Beziehungsgestaltung eintauchen}

$\mathrm{Zu}$ einer umfassenden Selbsterfahrungsexpedition gehört nicht nur ein ausführliches Erforschen des Selbstkonzeptes sowie ein längerer Aufenthalt in der Kindheit, sondern auch ein Durchforsten von Mustern und Interaktionsformen, die spätere Beziehungen prägen. Wie agiert man als ,role-giver“, wie als ,,role-receiver“ wenn es um Liebesbeziehungen geht, wie in Freundschaften oder wie, wenn es zu Konflikten kommt?

Hier kann es von Interesse sein, welche Verhaltensweisen gezeigt werden, wenn es zu Meinungsverschiedenheiten kommt. Aber zuerst sollen etwaige rigide Rollenkonfigurationen enttarnt werden. 


\subsection{Auf der Suche nach rigiden Rollenkonfigurationen}

Bei der Fragestellung, warum eine Person Liebesbeziehungen immer wieder mit Personen eingeht, bei denen sie nicht das bekommt, was sie sich wünscht, kann auf folgende Weise vorgegangen werden: Der*die Klient*in zeichnet sein*ihr soziokulturelles Atom mit jenen Rollen auf, die in Liebesbeziehungen zum Vorschein treten. Rund um das soziokulturelle Atom werden die Eigenschaften der derzeitigen und früheren Partner*innen aufgelistet. Nun wird analysiert, ob bestimmte Rollenkonfigurationen zwischen dem*der Klient*in und ihren Liebespartner*innen sich wiederholen. Welche sind diese? Welche Rolle im eigenen kulturellen Atom wird von dem angezogen, was die Partner*innen bieten? Welche Komplementärrollen der Partner*innen erfüllen bestimmte Bedürfnisse des*der Klient*in so stark und überschatten das, was ,im Gesamtpaket“ noch enthalten ist? Wie agiert man in diesen Beziehungsformen als role-giver, wie als role-receiver? Was würde es benötigen, um diese Muster zu durchbrechen?

In Protagonist*innenspielen kann erfasst werden, was hinter diesen speziellen Rollenkonfigurationen steht und in einer Zukunftsprobe, was benötigt wird, um die eingefahrenen Rollenkonfigurationen zu durchbrechen.

\subsection{Mit Hilfe innerer Anteile, perfekte Zielen entlarven}

Ein wie oben beschriebenes soziokulturelles Atom kann auch hilfreich sein, themenspezifische perfekte Ziele zu enttarnen. Unter perfekten Zielen versteht Schacht (2009, S. 92) Ziele, die eine Person um ,jeden Preis der Welt" erreichen möchte, deren Umsetzung aber ein unrealistisches Unterfangen ist, wie etwa von allen geliebt und wertgeschätzt zu werden. Unterschieden werden perfekte Annäherungsziele, die sich auf die Befriedigung bestimmter Bedürfnisse beziehen und Vermeidungsziele, bei denen versucht wird, aversiven Erfahrungen aus dem Weg zu gehen (Hintermeier 2018, S. 96). Schacht (2009, S. 100 f.) spricht von themenspezifischen perfekten Zielen, wenn sie nur in bestimmten Situationen in Erscheinung treten. Im Gegensatz dazu stehen strukturell bedingte perfekte Ziele, die mehr oder minder ständig in Interaktionen wirksam werden, da die Selbstregulationsfähigkeit der betroffenen Person nur über eine geringe Komplexität verfügt. Bei Selbsterfahrungsklient*innen geht man davon aus, dass themenspezifische perfekte Ziele nur in Ansätzen zu Tage treten und dass sie noch nicht zu einer Störung geführt haben. Typisch hierfür wäre im beruflichen Kontext aus Furcht vor Ablehnung, nicht „Nein sagen“ zu können. Hierzu ein Beispiel:

In einem soziokulturellen Atom einer Klientin wird in Verbindung mit ihrer beruflichen Tätigkeit eine besonders starke Verknüpfung mit ihren inneren Rollen als: „Everyone's Darling“, „Superwoman“ und die „Ständig Erschöpfte“ festgestellt. Dieser Zusammenhang wird in einem Protagonist*innenspiel unter die Lupe genommen. Dabei wird entdeckt, dass die Angst, an Beliebtheit zu verlieren und der Anspruch, als Superwoman alles schaffen zu müssen, sie davon abhält, zu bestimmten Aufgaben Nein zu sagen. Als Ursprungsszene dazu entpuppt sich der Liebesentzug der Mutter, der eintrat, wenn die Klientin 
als Kind die von ihr erwarteten Leistung nicht erbracht hatte. Im weiteren Verlauf besucht die Mutter im Sinne der Surplus-Reality des Psychodramas und dem ,wahren zweiten Mal“" eine Erziehungsberatung, bei der sie über die Folgen ihres Tuns aufgeklärt wird. Die ,geläuterte“ Mutter vermittelt ihrem Kind, das es auch geliebt wird, wenn es Fehler macht. Als Abschluss wird erkundet, welchen Einfluss eine imperfekte Zielsetzung ,Ich möchte meine Arbeit so gut wie möglich erfüllen, aber Fehler können passieren“ auf die Ausprägung der anderen Rollen hat.

\subsection{Die gepflegte Waffenkammer inspizieren}

Dies ist ein Arrangement, das ich bei Hildegard Pruckner kennen gelernt habe, die es in Anlehnung an Bücher von Christine Bauer-Jelinek (2020a, b) entwickelt hat. Hierbei geht es darum, die Stärken und Schwächen in Situationen zu erforschen, die Durchsetzungs- und Konfliktfähigkeit erfordern. Ziel ist, in Erfahrung zu bringen, zu welchen Konfliktmustern die Teilnehmenden neigen und welche Strategien bevorzugt eingesetzt werden, um sich durchzusetzen oder in Streitsituation behaupten zu können.

Begonnen wird mit einer Imagination, in der die Gruppenteilnehmer*innen sich verschiedene Konfliktsituationen vorstellen sollen. Wie reagieren sie in Liebesbeziehungen, wenn es zu Streitigkeiten kommt, wie innerhalb der Familie? Zu welchen Strategien greifen sie im beruflichen Kontext, wie gehen sie vor, wenn sie im Freundeskreis ihren Ansichten Gehör verschaffen wollen?

Im zweiten Schritt sollen sich die Teilnehmer*innen vorstellen, sie besäßen eine Waffenkammer. Die Waffen, die darin aufbewahrt wären, sollen grafisch dargestellt werden. Das kann zum Beispiel eine „Spitze Zunge“, ,ein Eisblock, der einen blitzschnell emotional erstarren lässt“, ,eine Tränendrüse“, „ein Pokerface“ u.v.m. sein.

Danach wird reflektiert, wann welche Waffen zum Einsatz kommen, wie sie angewendet werden, ob sie zum Ziel führen oder ob man sich damit in erster Linie ins eigene Fleisch schneidet. Ebenso soll erforscht werden, welche Waffen antiquiert sind und eingeschmolzen oder verschrottet gehören, mit welchen eventuell über das Ziel hinausgeschossen wird und welche Neuanschaffungen es geben könnte. Anregung hierfür kann bei anderen Waffenarsenalen gewonnen werden. Wichtig ist dabei darauf zu achten, ob man mit diesen Waffen selbst auch gut hantieren könnte.

\section{Durch Interaktion mit anderen mehr über sich selbst erfahren}

Das Rollenspiel eignet sich nicht nur in der Kindheit, um sich und seine Umwelt besser zu begreifen. Auch im Erwachsenenalter können Rollenspiele dazu dienen, Unbekanntes über sich zu erfahren und/oder neue Rollen auszuprobieren. Durch das spontane Eintauchen in eine Rolle und die lebendige Interaktion wird verhindert, dass das Handeln im Spiel zu sehr von der Ratio geprägt wird. Dadurch kann spontan etwas zum Vorschein treten, das bislang noch im Verborgenen gelegen ist. 


\subsection{Verborgene Schätze im Rollenspiel heben}

Typische psychodramatische Rollenspiele sind der „Psychodramazug“, eine „Zusammenkunft auf einem mittelalterlichen Markt“ oder „Gestrandet auf einer einsamen Insel“. Der Titel und der Inhalt des Spiels kann natürlich beliebig gewählt werden, wichtig ist, dass damit das Gruppenthema bearbeitet werden kann. Die Gruppenteilnehmer*innen sollen sich spontan für eine Rolle in diesem Spiel entscheiden, diese ausgestalten (Verkleidungen können dabei hilfreich sein) und mit anderen in Interaktion treten. Gewinnbringend ist es auch, nach einiger Zeit einen Rollentausch mit einer anderen Figur im Spiel vorzunehmen oder eine andere Rolle auszuprobieren.

Nach dem Rollenspiel ist es für die Teilnehmenden interessant zu reflektieren, wie es ihnen in den verschiedenen Rollen ergangen ist, welche als lustvoll und welche als herausfordernd erlebt wurden. Von Bedeutung ist auch, welche Verbindung sich mit der gewählten Rolle und dem derzeitigen Leben herstellen lässt und ob sie dabei Neues über sich in Erfahrung bringen konnten. Neigen sie eher dazu, Altbekanntes zu übernehmen oder Neues, Unbekanntes auszuprobieren?

In der Integrationsphase soll überlegt werden, welcher Nutzen aus einem Transfer der gemachten Erfahrungen ins normale Leben gezogen werden könnte.

\subsection{Ein Ausflug in die Welt der Märchen, Sagen und Mythen}

Eine besondere Form des Gruppenspiels stellt das Nachspielen von Märchen und Mythen sowie deren Neugestaltung dar. Nach Stadler (2015) kann das psychodramatische Märchenspiel sowie die Auseinandersetzung mit Szenen aus Sagen und Mythen zum besseren Verständnis „,von inneren Rollen, unbewussten Konflikten und anstehenden Lebensaufgaben“ (S. 9) beitragen. Der Verfremdungseffekt, der durch die Übernahme dieser archetypischen Rollen entsteht, kann die Motivation der Teilnehmenden erhöhen, ,sich auf schwierige Rollen und Emotionen einzulassen, wie der Neid im Mutter-Tochter-Konflikt in Schneewittchen" (Kunz Mehlstaub und Stadler 2018, S. 99). Das Märchen oder die Sage kann von dem*der Gruppenleiter*in vorgegeben sein oder von der Gruppe selbst gewählt werden. Im zweiten Fall ist interessant, welches Märchen oder Legende von der Gruppe ausgesucht wird. Ist dies ein Märchen, das einen oder eine Hauptdarsteller*in hat oder eines, in dem eine ganze Gruppe im Mittelpunkt steht, wie z.B. „Die Schildbürger“? Hier kann auch hinterfragt werden, wie sehr eine Person sich bei der Wahl des Märchens einbringt. Hält sie sich im Hintergrund, versucht sie zu vermitteln oder sich durchzusetzen.

Auskunft darüber, wie ein Gruppenmitglied von den anderen wahrgenommen und eingeschätzt wird, kann erhalten werden, wenn die Gruppe entscheidet, wer welche Rolle übernehmen soll. Dies kann auch in Zusammenhang mit einer Aufgabenstellung geschehen, wie „Teilt die Rollen des Märchens so auf, dass die jeweiligen Gruppenmitglieder die Rollen bekommen, von denen sie am meisten profitieren können“. Welche Überlegungen hinter den Rollenaufteilungen gestanden sein könnten, kann in der Integrationsphase erfragt werden. Im Sharing steht der Zusammenhang mit den in diesem Spiel gemachten Erfahrungen und dem realen Leben im Vordergrund. 


\section{Resümee}

In diesem Artikel wurden einige von mir erprobte psychodramatischen Arrangements vorgestellt, die bei den von mir angeleiteten Selbsterfahrungsreisen zum Einsatz kommen. Die Variantenvielfalt von psychodramatischen Arrangements, die ein Entdecken der eigenen Seelenlandschaft unterstützen, ist allerdings nahezu grenzenlos. In diesem Sinne kann ich jedem oder jeder empfehlen, der*die eine erlebnisorientierte, abwechslungsreiche und lustvolle Reise in die eigene Innenwelt unternehmen möchte, den Reiseveranstalter „Psychodrama“ zu wählen.

Funding Open access funding provided by Danube University Krems University for Continuing Education.

Open Access Dieser Artikel wird unter der Creative Commons Namensnennung 4.0 International Lizenz veröffentlicht, welche die Nutzung, Vervielfältigung, Bearbeitung, Verbreitung und Wiedergabe in jeglichem Medium und Format erlaubt, sofern Sie den/die ursprünglichen Autor(en) und die Quelle ordnungsgemäß nennen, einen Link zur Creative Commons Lizenz beifügen und angeben, ob Änderungen vorgenommen wurden.

Die in diesem Artikel enthaltenen Bilder und sonstiges Drittmaterial unterliegen ebenfalls der genannten Creative Commons Lizenz, sofern sich aus der Abbildungslegende nichts anderes ergibt. Sofern das betreffende Material nicht unter der genannten Creative Commons Lizenz steht und die betreffende Handlung nicht nach gesetzlichen Vorschriften erlaubt ist, ist für die oben aufgeführten Weiterverwendungen des Materials die Einwilligung des jeweiligen Rechteinhabers einzuholen.

Weitere Details zur Lizenz entnehmen Sie bitte der Lizenzinformation auf http://creativecommons.org/ licenses/by/4.0/deed.de.

\section{Literatur}

v. Ameln, F., Gerstmann, R. \& Kramer, J. (2005). Psychodrama. Berlin: Springer.

Bauer-Jelinek, C. (2020a). Die helle und die dunkle Seit der Macht. Wie sie ihre Ziele durchsetzen können, ohne ihre Werte zu verraten. Ecowin.

Bauer-Jelinek, C. (2020b). Die geheimen Spielregeln der Macht. Und die Illusion der Gutmenschen. Ecowin.

Bermann, B., \& Küster, H. (2019). Die innere Teamsitzung im Gruppensetting Das strukturierte Erkunden von drei Anteilen des inneren Teams in Form von schriftlichen Dialogen. Zeitschrift für Psychodrama und Soziometrie, 18, 103-108. https://doi.org/10.1007/s11620-019-00476-6.

Brüderl, L., Riessen, I., \& Zens, I. (2021). Therapie-Tools. Selbsterfahrung. Weinheim Basel: Beltz.

Döller, M. (2016). Die Mid-Career-Crisis als depressives Symptom - Axiodrama als methodischer Zugang. Zeitschrift für Psychodrama und Soziometrie, 15, 95-107. https://doi.org/10.1007/s11620-016-03206.

Hintermeier, S. (2018). Psychodramatische Störungstheorie und Veränderungsprozessmodell. In S. Kern \& S. Hintermeier (Hrsg.), Psychodrama-Psychotherapie im Einzelsetting. Theorie und Praxis im Monodrama (S. 92-107). Wien: facultas.

Kunz Mehlstaub, S., \& Stadler, C. (2018). Psychodrama-Therapie. Stuttgart: Kohlhammer.

Mösler, T., \& Poppek, S. (2016). Eine Einführung in das Thema Selbsterfahrung. In T. Mösler, S. Poppek, J. Kemper, A. Rose \& W. Dorrmann (Hrsg.), Der Blick auf sich selbst - Selbsterfahrung in der Psychotherapie (S. 11-30). Tübingen: Psychotherapie-Verlag.

Peichl, J. (2011). Jedes Ich ist viele Teile. Die inneren Selbst-Anteile als Ressource nutzen. München: Kösel.

Petzold, H. (1993). Integrative Therapie. Modelle, Theorien und Methoden für eine schulenübergreifende Psychotherapie. Paderborn: Junfermann.

Petzold, H., \& Mathias, U. (1982). Rollenentwicklung und Identität. Paderborn: Junfermann.

Schacht, M. (2003). Spontaneität und Begegnung. Zur Persönlichkeitsentwicklung aus der Sicht des Psychodramas. München: InScenario. 
Schacht, M. (2009). Das Ziel ist im Weg. Störungsverständnis und Therapieprozess im Psychodrama. Wiesbaden: VS.

Schnabel, K., v. Ameln, F., \& Stadler, C. (2019). Innere Anteile und lebendige soziale Teilhabe. Zeitschrift für Psychodrama und Soziometrie, 18, 7-22. https://doi.org/10.1007/s11620-019-00471-x.

Stadler, C. (2015). Traum und Märchen. Handlungsorientierte Psychotherapie. Stuttgart: Kohlhammer.

Stadler, C., \& Kern, S. (2010). Psychodrama. Eine Einführung. Wiesbaden: VS.

Wikipedia https://de.wikipedia.org/wiki/Selbstwert. Zugegriffen: 29. Okt. 2021.

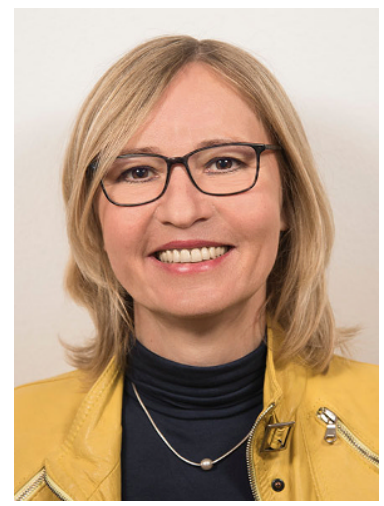

Sabine Kern 1966, Mag.a, MSc, Klinische Psychologin, Gesundheitspsychologin und Psychodrama-Therapeutin in freier Praxis. Lehrtherapeutin und Lehrsupervisorin im ÖAGG, an der Donau-UniversitätKrems und der Paris-Lodron-Universität Salzburg. Stellvertretende Fachsektionsleiterin der Fachsektion Psychodrama im ÖAGG. Redaktionsmitglied der Zeitschrift für Psychodrama und Soziometrie. 